NASA/CR-1998-208457

ICASE Report No. 98-30

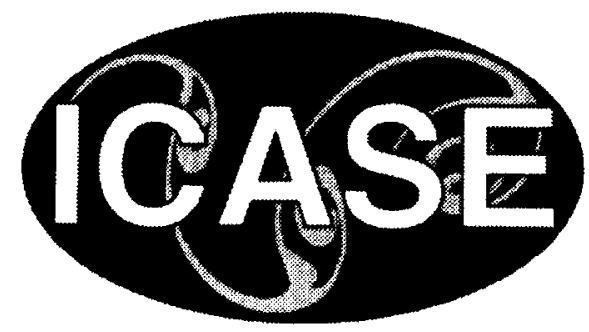

\title{
On the Propagation of Small Perturbations in Two Simple Aeroelastic Systems
}

Angelo Iollo

Politecnico di Torino, Torino, Italy

Manuel D. Salas

ICASE, Hampton, Virginia

Institute for Computer Applications in Science and Engineering NASA Langley Research Center

Hampton, VA

Operated by Universities Space Research Association

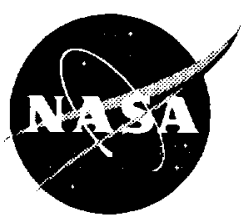

National Aeronautics and

Space Administration

Langley Research Center

Hampton, Virginia 23681-2199
Prepared for Langley Research Center under

Contract NAS1-19480 and NAS1-97046 
Available from the following:

NASA Center for AeroSpace Information (CASI) 7121 Standard Drive

Hanover, MD 21076-1320

(301) 621-0390
National Technical Information Service (NTIS)

5285 Port Royal Road

Springfield, VA 22161-2171

(703) 487-4650 


\title{
ON THE PROPAGATION OF SMALL PERTURBATIONS IN TWO SIMPLE AEROELASTIC SYSTEMS*
}

\author{
ANGELO IOLLO ${ }^{\dagger}$ AND MANUEL D. SALAS ${ }^{\ddagger}$
}

\begin{abstract}
In this paper we investigate the wave propagation patterns for two simple flow-structure problems. We focus on the study of the propagation speeds of the waves in the fluid and in the structure, as the rigidity of the structure and the Mach number of the undisturbed flow are changing. Some implications concerning the sound emission by inhomogeneities eventually present in the structure are discussed.
\end{abstract}

Key words. aeroelasticity

Subject classification. Physical Scicnces

1. Introduction. The motivations for this study are twofold. On the one hand we wanted to study the effects of the mean flow on the acoustic-waves speed, in the presence of a coupling with a structural element bounding the fluid. On the other hand, since the energy of a perturbation is partitioned between fluid and structure according to its speed, we were interested in investigating how the Mach number of the undisturbed flow may affect the noise scattered at inhomogencities by the structure.

In classical papers on acroelastic interactions, the time evolution of small perturbations is studied [4]. The stability boundaries are determined as functions of a speed parameter (the ratio of the wave velocity in the panel in absence of coupling and the wave length of the disturbance). It is also found that a panel characterized elastically by flexural forces only is unstable at any finite airspeed for sufficiently large wave lengths, whereas the introduction of membrane tension will lead to instability only for airspecds grater than to the minimum wave velocity of the panel. More recently the same problem was studied from another view point, the interest being the scattering of a bending wave by an inhomogeneity in an otherwise homogeneous and infinite pancl immersed in a fluid at rest [3]. Given a certain frequency of the perturbation, the dispersion relation of the coupled system is studied in terms of the wave number, whereas in the study of stability, a frequency analysis was preferred in order to detect the eventual time-wise growth of the propagating wave.

The study of the dispersion relation for a homogeneous beam is a preliminary step in analyzing the behavior of the air-beam system in the presence of inhomogeneities. In fact, the effect of gaps, stiffeners et cetera, is accounted by the presence, in the right hand side of the beam equation, of a linear combination of the Dirac function and its derivatives. The right hand side of the beam equation amounts to a forcing on the system whose response is, in the Fourier space, the ratio between the Fourier transform of the forcing term and the Fourier transform of the dispersion relation. Therefore, in the physical space, the solution is governed by the poles of such ratio, which are in turn the zeros of the dispersion relation.

In what follows we first studied a simple one-dimensional configuration in which the Mach number plays a role only on the stability bounds, while in the two-dimensional case the Mach number has an important effect on the solution of the dispersion relation, allowing or not certain waves to appear. The one-dimensional

* This research was supported by the National Acronautics and Space Administration under NASA Contract Nos. NAS119480 and NAS1-97046 while the authors were in residence at the Institute for Computer Applications in Science and Engineering (ICASE), NASA Langley Research Center, Hampton, VA 23681-2199.

${ }^{\dagger}$ Dipartimento di Ingegneria Acronautica c Spaziale, Politecnico di Torino, 10129 Torino, Italy. Present address: INRIA Sophia Antipolis, Projet SINUS, Sophia Antipolis Cedex, France.

$¥$ ICASE, NASA Langley Rescarch Center, Hampton, VA 23681-2199. 
case, however, has the merit of showing clearly the influence of the fluid-structure coupling on the speed of the propagating waves. We may anticipate that for low values of the stiffness, the propagation speeds in the beam and in the fluid are remarkably different from those in the uncoupled case.

2. Quasi One-Dimensional Coupling. We study the flow of a compressible fluid through a nozzle with elastic walls. The nozzle walls are loaded by the pressure difference between an outside ambient pressure and the local internal fluid pressure. The flow is assumed to be quasi one-dimensional, inviscid and isentropic. Under these hypothesis the non-dimensional equations governing the flow are the following

$$
\begin{array}{r}
\frac{2}{\gamma-1} c_{t}+c u_{x}+\frac{2}{\gamma-1} c_{x} u+\frac{c}{H}\left(H_{t}+u H_{x}\right)=0 \\
u_{t}+u u_{x}+\frac{2}{\gamma-1} c c_{x}=0
\end{array}
$$

where $c$ is the local speed of sound, $u$ is the velocity of the fluid, $H$ the nozzle hight and $\gamma$ the specific heats ratio.

In addition, we assume that the deformation of the walls of the nozzle are so small that the motion is governed by the linear beam equation

$$
m H_{t t}+D H_{x x x x}=p_{i}-p_{0}
$$

where $D$ is the bending stiffness, $p_{i}$ the local pressure of the fluid, $p_{0}$ is the outside ambient pressure and $m$ the linear mass of the walls that is 1 in what follows.

The coupling between the quasi one-dimensional fluid equation and the beam equation, which is due to the pressure difference on the right hand side of eq. 2.3 , is interesting because of the different nature of the partial differential equations (PDEs) governing the fluid and the nozzle wall motion. If we consider only the fluid, we have a hyperbolic system of PDEs representing signals that propagate on two characteristics with speeds $u \pm c$. The perturbations are felt in the fluid only after a finite time, needed for the perturbation to propagate from the source to the receiver. On the other hand, the linear beam equation is parabolic, i.e., perturbations are immediately felt all along the beam, although the phenomena is still evolving in time. In fact, from the dispersion relation of this PDE we have two waves traveling with speeds $\pm \sqrt{D} k$ and two near fields [1].

The coupled system is parabolic, but the traveling waves of each uncoupled system play an important role for what concerns the stability of the solution and the partition of the energy of the perturbations between the fluid and the nozzle walls.

Let us consider a nozzle with straight walls at $t=0$ and with an inlet Mach number $M_{0}$. We want to study the evolution of small perturbations for this system. Take $c=c_{0}+c^{\prime}, u=u_{0}+u^{\prime}$ and $H=H_{0}+H^{\prime}$ and substitute in eqs. 2.1- 2.2 and 2.3. Assuming that $p_{0}=\rho_{0}=1$, that the prime quantities are small, and dropping the prime notation, we obtain the following system for the perturbations

$$
\begin{array}{r}
\frac{2}{\gamma-1} c_{t}+c_{0} u_{x}+\frac{2}{\gamma-1} u_{0} c_{x}+\frac{c_{0}}{H_{0}}\left(H_{t}+u_{0} H_{x}\right)=0 \\
u_{t}+u_{0} u_{x}-\frac{2}{\gamma-1} c_{0} c_{x}=0 \\
H_{t t}+D H_{x x x x}-\frac{2}{\gamma-1} c_{t}\left(\frac{c_{0}^{2}}{\gamma}\right)^{\frac{1}{\gamma-1}} c=0
\end{array}
$$

This system of PDEs governs the evolution of small disturbances in a nozzle with parallel elastic walls. Assuming that the solution has the form

$$
c=\hat{c} e^{i(k x-\omega t)}
$$




$$
\begin{aligned}
u & =\hat{u} e^{i(k x-\omega t)} \\
H & =\hat{H} e^{i(k x-\omega t)}
\end{aligned}
$$

we substitute in eqs. 2.4-2.6 to get

$$
\begin{aligned}
& \hat{u}=\frac{2}{\gamma-1} \frac{c_{0} k}{\omega-u_{0} k} \hat{c} \\
& \hat{H}=\frac{2}{\gamma-1} c_{0}\left(\frac{c_{0}^{2}}{\gamma}\right)^{\frac{1}{\gamma-1}} \frac{\hat{c}}{D k^{4}-\omega^{2}} \\
& \left(\omega-u_{0} k\right)^{2}\left[1+\frac{c_{0}^{2}}{H_{0}\left(D k^{4}-\omega^{2}\right)}\left(\frac{c_{0}^{2}}{\gamma}\right)^{\frac{1}{\gamma-1}}\right]-c_{0}^{2} k^{2}=0
\end{aligned}
$$

Note that as $D \rightarrow \infty$ or $k \rightarrow \infty$ the system becomes increasingly uncoupled, i.e., the evolution of the perturbations in the beam are less and less influenced by the presence of the fluid and vice versa.

For given wave number $k$, we may solve eq. 2.12 with respect to $\omega$. When $\operatorname{Im}(\omega) \neq 0$ the corresponding mode of oscillation is unstable. Figure 2.1 shows a plot of $\operatorname{Re}(\omega / k)$ with respect to $D$ when $M_{0}=0$. The four solutions are obviously real and symmetric with respect to the abscissa. No unstable solution is possible since there is no forcing on the system.

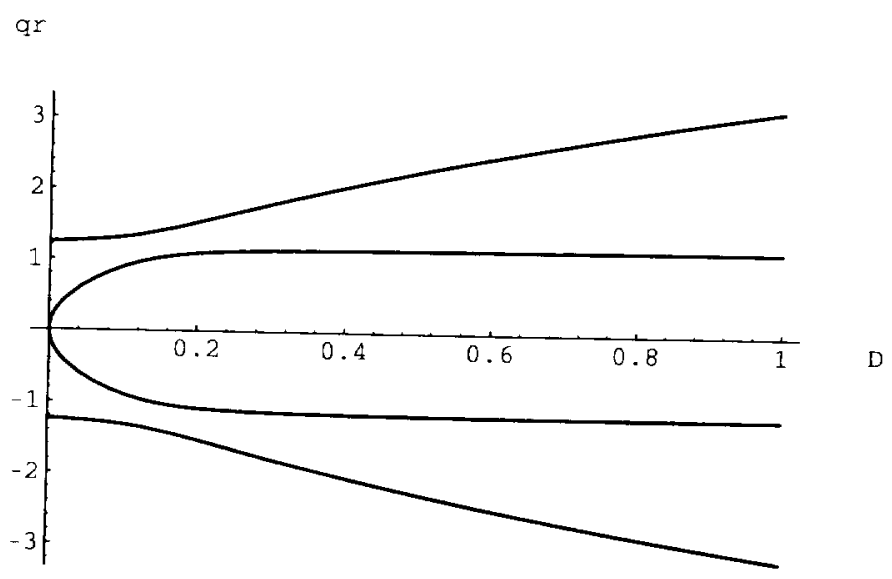

FIG. 2.1. Solutions of the dispersion relation for a quasi one-dimensional coupling. $c_{0}=\sqrt{1.4}, M_{0}=0, k=\pi$ and $q r=R e(\omega / k)$. The branches of the solution are named $a, b, c, d$ from top to bottom.

The four solutions represent waves which travel in the positive and negative direction of the $\mathrm{x}$-axis. They correspond to the waves present in each of the uncoupled systems which have speeds $\pm c_{0}$ and $\pm \sqrt{D} k$. Solutions $b$ and $c$ go to 0 when the stiffness is zero (no wall scparating the ambient and internal flow), while the waves corresponding to solutions $a$ and $d$ have speeds equal to that of the signals in the fluid $\pm c_{0}$. For increasing stiffness the solutions gradually shift role. For example, the solutions that are 0 for $D=0$ asymptotically approach the value of $\pm c_{0}$ when the system becomes uncoupled, i.e., for $D \rightarrow \infty$. Conversely, $a$ and $d$ approach the curves $\omega / k= \pm \sqrt{D} k$ as $D \rightarrow \infty$. 
The partition of the energy of the perturbations between beam and fluid depends on the phase speed $\omega / k$, of the wave considered. It is seen from eq. 2.11 that for a given amplitude $\hat{c}$, if the speed of the wave considered is close to $\pm \sqrt{D} k$ then $\hat{H} \rightarrow \infty$. This means that when the speed of a wave in the coupled system is close to the speed of a wave present, for example, in an isolated beam, the energy of the perturbation is mainly concentrated in the beam. Similar arguments can be made for waves whose energy is mainly in the fluid.

$q r$

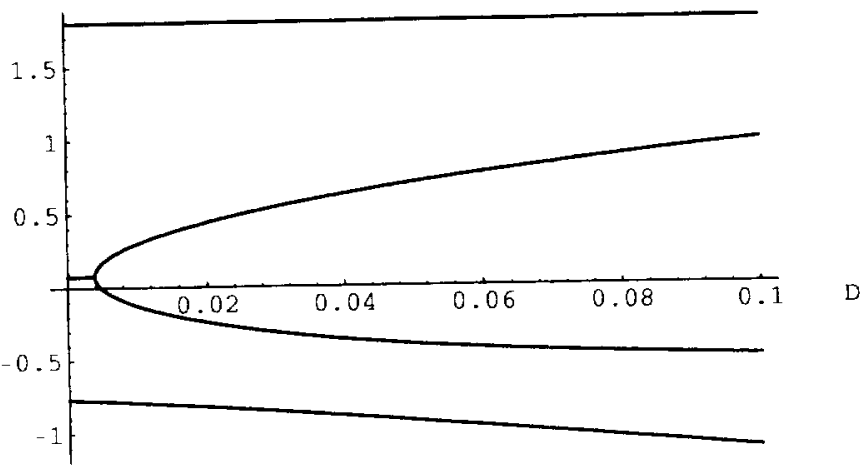

i)

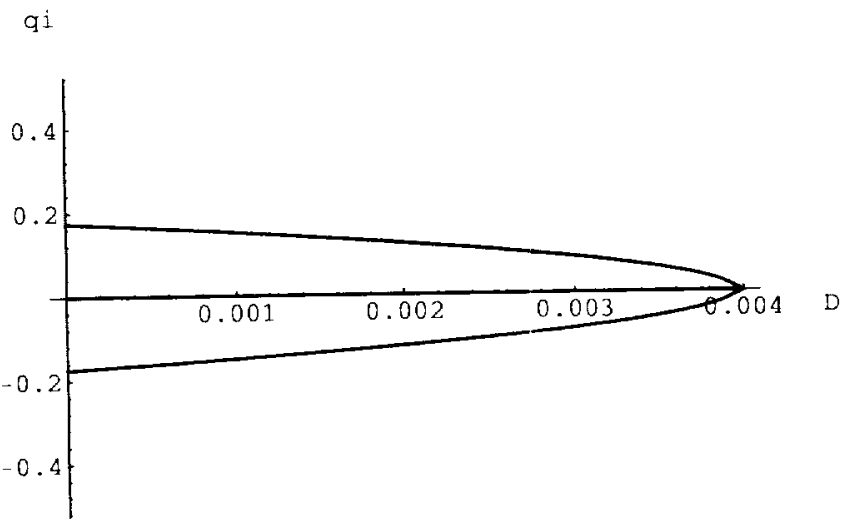

ii)

FIG. 2.2. Solutions of the dispersion relation for a quasi one-dimensional coupling. $c_{0}=\sqrt{1.4}, M_{0}=0.5, k=\pi$ i) $q r=\operatorname{Re}(\omega / k)$, ii) $q r=\operatorname{Im}(\omega / k)$. The branches of the solution are named $a, b, c, d$ from top to bottom of figure i).

In fig. 2.2, we illustrate the case corresponding to $M_{0}=0.5$. Now we find that there is a range of values of $D$ where $\operatorname{Im}(\omega / k) \neq 0$ for the solutions $b$ and $c$. The existence of this region indicates that unstable motion can be triggered by small disturbances with given wave number. Note that $\operatorname{Im}(\omega / k) \neq 0$ corresponds to the 
small region in fig. 2.2 where the branches b and c collapse into one curve, i.e., the speed of propagation of the two waves is the same. This is necessarily the case since the dispersion relation is a fourth order polynomial in $\omega$. Note also that the solutions $b$ and $c$ have asymptotes $c_{0}(0.5 \pm 1.0)$.

Because the unstable modes are associated with the collapsed branches $b$ and $c$, we can conclude that their energy is mostly in the beam. Interestingly, there is a range of values for the stiffness for which the unstable modes can propagate only in the positive direction.

2.1. Computational experiment. We considered a simply supported beam of unit length which is in contact with a fluid at rest governed by eqs. 2.1-2.2 on one side, and to a constant ambient pressure equal to that of the unperturbed fluid on the other side. We took a simply supported beam so that there are no near fields generated at the boundaries [1]. The flow takes place between the elastic beam and a rigid wall. This elastic "hose" connects two reservoirs whose pressure is kept constant and equal to that of the unperturbed flow in the hose. Therefore, the boundary points are nodal points for the pressure and displacement waves as well. When the beam is displaced from its equilibrium position it will perform free periodic oscillations corresponding to a superposition of the modes excited by the initial condition. There is no dissipative external force acting on the system and the system is conservative.

The beam equation was solved by mean of a semi-discretization based on a Galerkin projection of the solution on the eigenmodes of an isolated simply supported beam. This results in the solution of a set of ordinary differential equations (ODEs) for cach mode taken into account. The ODEs are then integrated in time by means of a standard fourth order Runge-Kutta scheme. Besides providing high resolution, this approach allows us to control very closely the modes of the coupled system excited by the initial condition which drives the system out of equilibrium. The given initial condition is the beam displacement. In particular, we displace the beam so that only the first mode of oscillation has non-null amplitude, i.c., $H(x, 0)=h \sin \pi x$ with small $h$. Thus, we are able to impose the wave number of the free oscillations in order to compare the frequencies resulting from the simulation with that computed by eq. 2.12. Other modes of oscillation have amplitudes of much lower order compared to that excited.

The fluid equations are discretized by a finite-volume scheme where the fluxes at the volume interfaces are computed as in [5]. Higher order accuracy is achieved by means of an ENO algorithm, see [2]. The number of computational volumes used to discretize the flow equations is 1000 , so that the accuracy of the results is of the order of $10^{-6}$. The computations where run in double precision.

In fig. 2.3 we plot the Mach number at the inlet of the nozzle versus time. It is seen that two frequencies of oscillation are present. Becausc of the set up of the experiment, the perturbation is not traveling, but forming a standing wave in the nozzle (standing waves comprise traveling waves in both directions). The two frequencies of fig. 2.1 are the ones found in this experiment. In particular it was verified that the periods $T=2 \omega / \pi$ computed by eq. 2.12 with $D=0.001(1.61,21.1)$ are to a good approximation equal to those obtained with the numerical simulation $(1.64,21.7)$.

3. Two-Dimensional Coupling. Let us consider a two-dimensional case in which the equation governing the flow is the linear potential equation

$$
\left(1-M_{0}^{2}\right) \Phi_{x x}+\Phi_{y y}-\frac{1}{c_{0}^{2}}\left(2 U_{0} \Phi_{x t}+\Phi_{t t}\right)=0
$$

where $\left(\Phi_{x}, \Phi_{y}\right)=(u, v)$ are the components of the flow velocity vector, $\left(U_{0}, 0\right)$ and $c_{0}$ are, respectively, the velocity and speed of sound of the unperturbed flow, and $M_{0}=U_{0} / c_{0}$. 


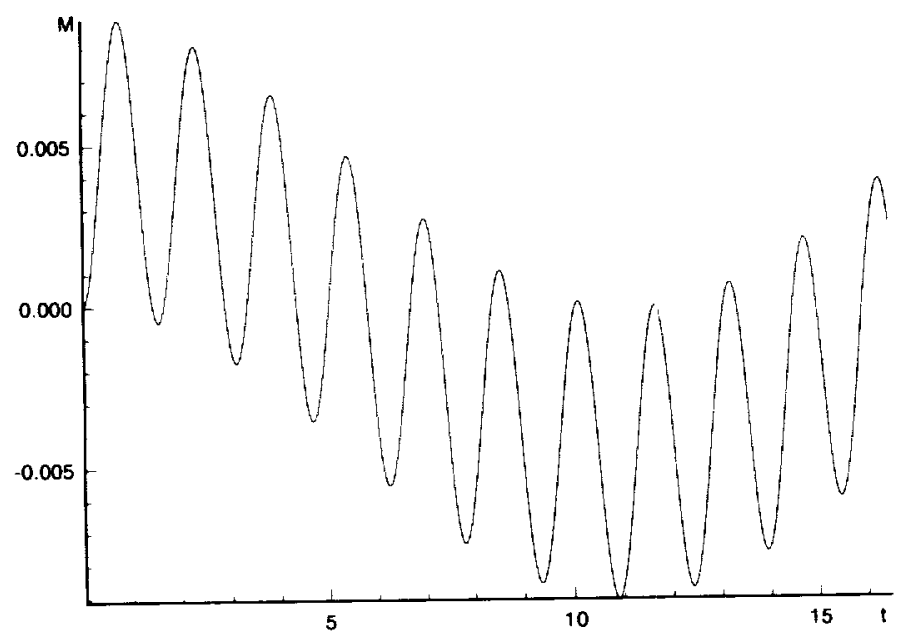

FiG. 2.3. Mach number versus time at nozzle inlet. $D=0.001$ and $k=\pi$.

Consider an infinitely long flexible surface separating two regions of the flow. On this surface the boundary condition on the flow is given by the equation

$$
\Phi_{y}=H_{t}+U_{0} H_{x}
$$

where $H$ is the distance of the flexible surface to the x-axis. In addition the potential $\Phi$ is required to vanish in the far field.

In the idealized system that we want to study we assume that the infinite surface is elastic and satisfies the linear small perturbation, beam equation

$$
H_{t t}+D H_{x x x x}=[p]=2 \rho_{0}\left(\Phi_{t}+U_{0} \Phi_{x}\right)
$$

where $\rho_{0}$ is the fluid density of the unperturbed flow and $[p]$ is the pressure jump across the wall. For simplicity, we are assuming that the flexible surface is wetted by the fluid on both sides. The case corresponding to a flow at rest on one side leads to more complex algebraic manipulations, but the conclusions would not be altered.

Equations 3.1-3.3 form a coupled system. The coupling comes about through the acrodynamic load on the moving surface (beam) and the boundary condition, cq. 3.2 .

We limit our study to such a linear model since we are interested in studying how the coupling affects the propagation of small amplitude waves. To do that, we take

$$
\Phi=\hat{\Phi} \exp \left[i\left(k_{1} x+k_{2} y-\omega t\right)\right]
$$

$$
H=\hat{H} \exp \left[i\left(k_{1} x-\omega t\right)\right]
$$

and we substitute these expressions in eqs. 3.1-3.3. The angular velocity $\omega$ is supposed to be a real number. Therefore, we consider waves whose amplitude are not diverging or decaying in time. 
Solving for $k_{2}$ in eq. 3.1 we obtain

$$
k_{2}=\sqrt{\left(\frac{\omega}{c_{0}}-M_{0} k_{1}\right)^{2}-k_{1}^{2}}
$$

Note that to have a finite amplitude wave for large $y$ and to ensure the radiation condition, i.e., outgoing waves in the far ficld, $k_{2}$ is either a positive imaginary or a positive real:

$$
k_{2} \in i R^{+} \text {or } k_{2} \in R^{+}
$$

These conditions are a very important discriminant for admitting or not certain solutions and we will make use of them later.

From eq. 3.2, we have

$$
\hat{\Phi}=-\frac{\omega-U_{0} k_{1}}{k_{2}} \hat{H}
$$

and from eq. 3.3 we obtain

$$
\left(D k_{1}^{4}-\omega^{2}\right) \hat{H}=-2 \rho_{0} i\left(\omega-U_{0} k_{1}\right) \hat{\Phi}
$$

Substituting cqs. 3.6 and 3.8 into eq. 3.3, and making use of eqs. 3.4 and 3.5 we obtain the dispersion relation for the coupled aeroclastic system.

The dispersion relation is nondimensionalized with respect to $K_{0}=\left(\omega^{2} / D\right)^{1 / 4}$ which is the wave number of the small perturbations traveling in the beam when there is no coupling with the fluid. Introducing also $k_{0}=\omega / c_{0}, \mu=k_{0} / K_{0}, K=k_{1} / K_{0}$ and $\nu=2 \rho_{0} / K_{0}$, the dispersion relation is written

$$
K^{4}-1=\frac{i \nu}{\sqrt{\left(\mu-\overline{\left.M_{0} K\right)^{2}-K^{2}}\right.}}
$$

The parameter $\mu$ has a physical meaning similar to that of the Mach number: it is the ratio between the speed of the perturbations in the beam to that in air when there is no coupling.

This equation relates the wave numbers and the frequencies of the small amplitude waves which can propagate in the coupled acroclastic system. In the case of $M_{0}=0$ the above equation reduces to

$$
K^{4}-1=\frac{\nu}{\sqrt{K^{2}-\mu^{2}}}
$$

which is identical to the dispersion relation obtained in [3], eq. 3.9, for a case with zero mean flow. Notice that $k_{2} / K_{0}=\sqrt{\left(\mu-M_{0} K\right)^{2}-K^{2}}$ which is the denominator of the right hand side of eq. 3.10 .

Let us consider now the uncoupled system, where the beam vibration is not affecting the perturbations in the fluid and vice versa. In this case, the nondimensional dispersion relation is

$$
K_{b}^{4}-1=0
$$

with solutions

$$
K_{b}= \pm 1, \pm i
$$

The solutions $K_{b}= \pm 1$ correspond to wave motion in the positive and negative directions of the $\mathrm{x}$-axis. The solutions $K_{b}= \pm i$ represent near ficlds generated close to some boundary, thesc are used to accommodate the boundary conditions if present. 
In the fluid, the acoustic waves propagating in the $x$ direction have speed $\omega_{f} / k_{f}=U_{0} \pm c_{0}$, from which we can compute the dimensionless wave number

$$
K_{f}=\frac{\mu}{M_{0} \pm 1}
$$

If we assume that the solutions of the coupled system are not very far from those of the uncoupled system eqs. 3.13 and 3.14 , we can make eq. 3.10 approximately solvable in closed form. Consider first the roots $K \approx \pm 1$ and the case $\left|\mu \pm M_{0}\right|<1$, after substituting in the right hand side of eq. 3.10 we have

$$
K^{4}-1=\frac{\nu}{\sqrt{1-\left(\mu \mp M_{0}\right)^{2}}}
$$

where we took into account the conditions 3.7. To the same order of approximation the solution of the above equation can be written

$$
K=1+\frac{\nu}{4 \sqrt{1-\left(\mu \mp M_{0}\right)^{2}}}
$$

Similarly $\left|\mu \pm M_{0}\right|>1$, we have

$$
K=1+\frac{i \nu}{4 \sqrt{\left(\mu \mp M_{0}\right)^{2}-1}}
$$

These waves are equivalent to the waves that in a isolated beam travel from $-\infty$ to $+\infty$ without attenuation. In the coupled case, depending on $\mu \pm M_{0}$, we have two different behaviors. For $\left|\mu \pm M_{0}\right|<1$, the wave number in the direction of $\mathrm{x}$-axis is real, while $k_{2} / K_{0} \in i R^{+}$, i.e., the wave is decaying in the direction of the $y$-axis, and therefore, since there is no energy radiated away, it propagates without attenuation in the direction of the $\mathrm{x}$-axis.

When $\left|\mu \pm M_{0}\right|>1, K$ has a non zero imaginary part. The wave number in the direction of the $y$-axis is real, i.e., energy is radiated away from the vibrating beam and therefore the wave is decaying as it propagates along the beam.

The equivalent of the near fields cxisting in the uncoupled beam are found when $K \approx \pm i$

$$
K= \pm i\left(1+\frac{i \nu}{4 \sqrt{\left(\mu \mp M_{0} i\right)^{2}+1}}\right)
$$

which is valid for any value of $\mu \pm M_{0}$, therefore the type of solution found for the coupled aeroelastic system is basically the same as for the near fields corresponding to $M_{0}=0$.

The solutions corresponding to the acoustic waves are found rewriting eq. 3.10 as

$$
\sqrt{\left(\mu-M_{0} K\right)^{2}-K^{2}}=\frac{i \nu}{K^{4}-1}
$$

then assuming $K \approx \mu /\left(M_{0} \pm 1\right)$, we have

$$
\sqrt{\left(\mu-M_{0} \frac{\mu}{M_{0} \pm 1}\right)^{2}-K^{2}}=\frac{i \nu}{\left(\frac{\mu}{M_{0} \pm 1}\right)^{4}-1}
$$

The above equation has acceptable solutions, in the sense of the conditions 3.7 , if and only if $\left|\mu /\left(M_{0} \pm 1\right)\right|>1$ which is equivalent to $\left|\mu \pm M_{0}\right|>1$. In this case the solutions are

$$
K=\frac{\mu}{M_{0} \pm 1}+\frac{\nu}{2\left[\left(\frac{\mu}{M_{0} \pm 1}\right)^{4}-1\right]} \frac{M_{0} \pm 1}{\mu\left[\left(1-M_{0}^{2}\right)+M_{0} \mu\right]}
$$


which are real numbers and therefore the waves travel without attenuation. The correspondent wave number in the direction of the $y$-axis is a pure imaginary, so there is no radiation of energy to infinity. These waves are the equivalent of the acoustic waves in the fluid for the uncoupled system.

It should be noted that there are as many different kinds of waves as there are different systems interacting, and that the particular wave with its velocity near that of one of the component systems will entrust its energy chicfly to that component. This can be secn by substituting the solutions of the dispersion relations into eq. 3.8, or eq. 3.9, and solving for the ratio of the amplitudes.

Compared to the case in which $M_{0}=0$, there is a reacher variety of solutions available, according to the inequality satisfied by $\mu \pm M_{0}$. In fact depending on the direction we consider, we may have either $\mu+M_{0}>1$ or $-\mu+M_{0}<1$. In this case for example, the last pair of solutions obtained would propagate only in the positive direction of the $\mathrm{x}$-axis.

This result is reasonable if we consider that what is important is the relative motion of the fluid with respect to the waves traveling in the beam, in this sense, it is interesting to compare to the results in [3] where a similar analysis is done for $M_{0}=0$. In this casc it is known that waves propagating in the beam in the $x$ direction radiate energy in the $y$ direction only if the wave is supersonic, i.e., $|\mu|>1$. When $M_{0} \neq 0$, we take a frame of reference at rest with respect to the fluid. In the relative motion, the speed of the wave in the beam is $\mu \pm M_{0}$.

Why these results are relevant to the noise emission from a rib stiffener? Intuitively it is clear that when the wave energy is mostly into the fluid, very little energy is scattered at the stiffener, while if the wave energy is mostly concentrated in the beam, the noise emission will be higher. This argument can be made rigorous if we consider that the eigenvalues of the free acroclastic system become the poles of the transfer function for the forced system constituted by the fluid, the beam and the stiffener. The number and the position of these poles in the complex plane now are function not only of $\mu$ but of $M_{0}$ as well. Therefore the emission of noise as a function of $\mu$, as for example presented in [3], depends now on the free stream Mach number.

\section{REFERENCES}

[1] L. Cremer, M. Heckl, and E.E. Ungar, Structure-Borne Sound, Springer Verlag, 1973.

[2] A. Harten, B. Engquist, S. Osher, and S.R. Chakravarthy, Uniformely high order accurate essentially non-oscillatory schemes iii, JCP, 71, 1987, pp. 231-303.

[3] M.S. Howe, Attenuation and Diffraction of Bending Waves at Gaps in Fluid Loaded Plates, IMA Journal of Applied Mathematics, 36, 1986, pp. 247-262.

[4] J.W. MiLes, On the aerodynamic instability of thin plates, Journal of the Aeronautical Sciences, No. 23, 1956, pp. 771-780.

[5] M. PANDOLFI, Contribution to the numerical prediction of unsteady fiows, AIAA Journal, 22, 1984, pp. 602-610. 


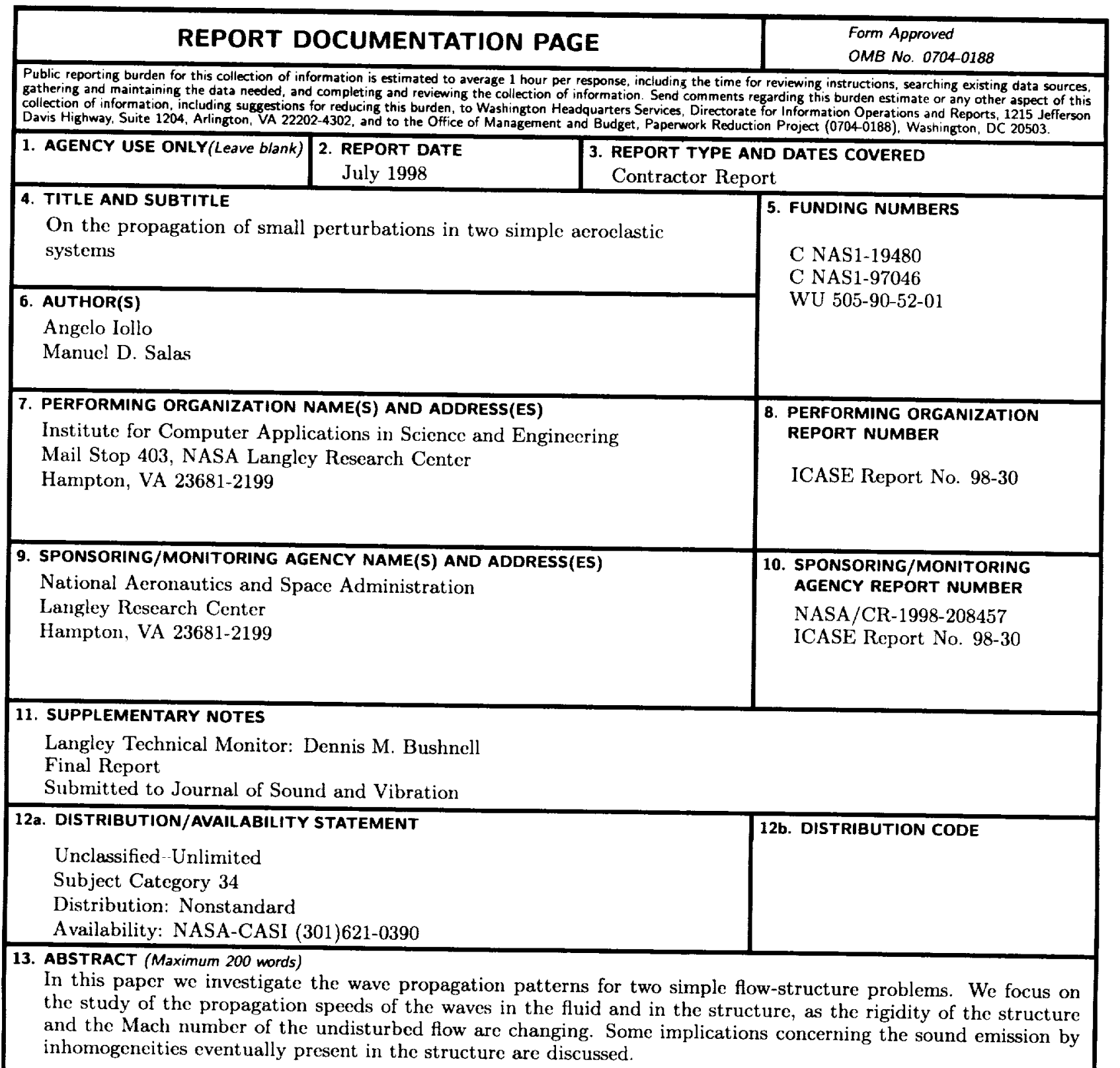

